\title{
Medium Access Control for Underwater Acoustic Sensor Networks with MIMO Links
}

\author{
Li-Chung Kuo \\ Wireless Networks and Embedded Systems \\ Laboratory \\ Department of Electrical Engineering \\ State University of New York at Buffalo \\ Buffalo, New York 14260 \\ Iku02@eng.buffalo.edu
}

\author{
Tommaso Melodia \\ Wireless Networks and Embedded Systems \\ Laboratory \\ Department of Electrical Engineering \\ State University of New York at Buffalo \\ Buffalo, New York 14260 \\ tmelodia@eng.buffalo.edu
}

\begin{abstract}
The requirements of multimedia underwater monitoring applications with heterogeneous traffic demands in terms of bandwidth and end-to-end reliability are considered in this article. To address these requirements, a new medium access control protocol named UMIMO-MAC is proposed. UMIMO-MAC is designed to i) adaptively leverage the tradeoff between multiplexing and diversity gain according to channel conditions and application requirements, ii) select suitable transmit power to reduce energy consumption, and iii) efficiently exploit the UW channel, minimizing the impact of the long propagation delay on the channel utilization efficiency.

To achieve the objectives above, UMIMO-MAC is based on a two-way handshake protocol. Multiple access by simultaneous and co-located transmissions is achieved by using different pseudo orthogonal spreading codes. Extensive simulation results show that UMIMO-MAC increases network throughput, decreases channel access delay, and decrease energy consumption compared with existing Aloha-like MAC protocols for UW-ASNs.
\end{abstract}

\section{Categories and Subject Descriptors}

C.2.2 [Computer-Communication Networks]: Network Protocols

\section{General Terms}

Design, Performance

\section{Keywords}

Underwater acoustic sensor networks, Medium access control, Multiple input multiple output

\section{INTRODUCTION}

Multimedia underwater sensor networks [1] would enable new applications for underwater multimedia surveillance, undersea explorations, video-assisted navigation and environmental monitoring. However, these applications require much higher data rates

Permission to make digital or hard copies of all or part of this work for personal or classroom use is granted without fee provided that copies are not made or distributed for profit or commercial advantage and that copies bear this notice and the full citation on the first page. To copy otherwise, to republish, to post on servers or to redistribute to lists, requires prior specific permission and/or a fee.

MSWiM'09, October 26-29, 2009, Tenerife, Canary Islands, Spain.

Copyright 2009 ACM 978-1-60558-616-9/09/10 ...\$10.00. than currently available with acoustic technology, and more flexible protocol design to accommodate heterogeneous traffic demands in terms of bandwidth, delay, and end-to-end reliability.

To accommodate such traffic demands, we propose to leverage the potential of multiple-input-multiple-output (MIMO) transmission techniques on acoustic links, and develop a new cross-layer medium access control (MAC) protocol to flexibly exploit the potential performance increase offered by MIMO links under the unique challenges posed by the underwater environment.

The MIMO transceiver technology, also known as smart antenna technology, has attracted considerable attention in radio-frequency (RF) communications [29]-[27]. Instead of mitigating the impact of multipath fading, MIMO systems can exploit rich scattering and multipath fading to provide higher spectral efficiencies without increasing power and bandwidth. Hence, MIMO technology has the potential to take advantage of the rich scattering and multipath of the underwater acoustic environment to increase data transmission rates and improve link reliability.

This idea has also been recognized by the underwater acoustic communication community in recent years. In [23], [12], and [15], the feasibility of MIMO systems and related spatial coding and modulation was tested for underwater acoustic communications and a significant performance improvement was demonstrated compared with the conventional SISO system architecture. However, the existing literature focuses mostly on experimental study [23]-[24], with very limited previous work on system performance analysis [31]. Importantly, no previous effort has studied the impact of MIMO transceivers on the design of higher-layer communication protocols.

For these reasons, the objective of this paper is to explore the capabilities of underwater MIMO links, and to leverage these from the perspective of higher layer protocols, and in particular at the medium access control layer, with a cross-layer design approach. In particular, in this work:

1. We identify how the capabilities of MIMO links, in particular the tradeoff between transmission data rate and link error probability, impact MAC protocol design in UW-ASNs;

2. We develop a new MAC layer protocol called UMIMO-MAC that leverages MIMO capabilities. In particular, UMIMOMAC is fully distributed and relies on lightweight message exchange. Moreover, following a cross-layer design approach, UMIMO-MAC adapts its behavior to the condition of environmental noise, channel, and interference to maximize the network throughput or minimize the energy consumption, according to the Quality of Service (QoS) requirements of the traffic being transmitted; 
3. We show how the principles that UMIMO-MAC is based upon can constitute basic building blocks to provide differentiated levels of QoS in underwater networks, and advance in the direction of studying feasibility, limits, and solutions to transport multimedia traffic in underwater networks.

We emphasize that, to the best of our knowledge, our work constitutes the first research effort to develop higher-layer communication protocols for underwater networks with MIMO links.

The remainder of this paper is organized as follows. In Section 2, we review recent literature on MAC layer protocols for underwater acoustic networks and on protocol design for wireless networks with MIMO links. In Section 3, we introduce an underwater acoustic MIMO transceiver model. In Section 4, our proposed MAC protocol named UMIMO-MAC is introduced. In Section 5, we assess the performance of the proposed solutions through simulation experiments. Finally, in Section 6, we draw the main conclusions.

\section{RELATED WORK}

Apart from studies concerned with acoustic communications at the physical layer [14][25], recent research has concentrated on developing solutions at the medium access control (MAC) and network layers of the protocol stack. In [19], we proposed UW-MAC, a distributed MAC protocol tailored for UW-ASNs, which aims at achieving high network throughput, low channel access delay, and low energy consumption. UW-MAC is a transmitter-based codedivision multiple access (CDMA) scheme that incorporates a novel closed-loop distributed algorithm to set the optimal transmit power and code length. In [16], Slotted FAMA, a protocol based on a channel access discipline called floor acquisition multiple access (FAMA), which combines both carrier sensing (CS) and a dialogue between the source and receiver prior to data transmission, is proposed. In [13], a hybrid medium access control protocol for underwater networks is proposed, which includes a scheduled portion to eliminate collisions and a random access portion to adapt to changing channel conditions.

In [20], we investigated the problem of data gathering in a threedimensional network architecture at the network layer by considering interactions between the routing functionality and the characteristics of the underwater acoustic channel. Two distributed routing algorithms were introduced for delay-insensitive and delaysensitive applications. In [18], the cross-layer interactions between the solutions in [19] and [20] are studied. In [9], tradeoffs in the design of energy efficient routing protocols for underwater networks are studied. In particular, an analysis is conducted to show the strong dependence of the available bandwidth on the transmission distance, which is a peculiar characteristics of underwater environment [26]. Furthermore, the paper studies the relationship between the energy consumption of acoustic modems in various modes, i.e., transmit, receive and idle, which is different than that of terrestrial radio transceivers. Other significant recent studies on underwater networks have considered delay-reliability tradeoff analysis for underwater networks [32] and the benefits achievable from cooperative communications [3].

Previous work has focused on developing MAC protocols for terrestrial wireless ad hoc networks with MIMO links. In particular, in [28], centralized and distributed MAC protocols for ad hoc networks with MIMO links called Stream-Controlled Medium Access (SCMA) are proposed. Throughput is increased by simultaneously transmitting multiple independent data streams on the same channel. In [17], a MIMO MAC protocol for ad hoc networks named MIMOMAN is proposed. The network throughput is enhanced by allowing simultaneous multiple communications at

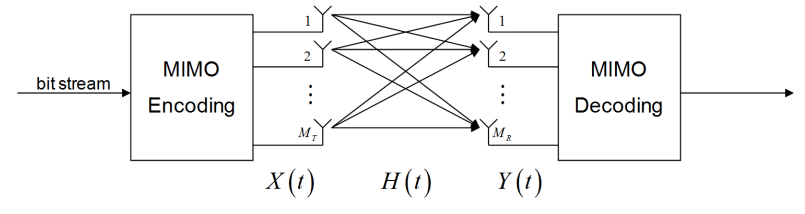

Figure 1: Underwater acoustic MIMO communications between a transmitter and a receiver

a higher data rate. However, the protocols in [28] and [17] do not consider the requirements of multimedia traffic, and consequently are not designed to leverage the multiplexing-diversity tradeoff typical of MIMO systems. In [11], a MAC protocol for MIMO ad hoc networks that considers the effect of spatial correlation on the system throughput is proposed. Links contend for the channel sequentially and then transmit data packets simultaneously. Stations use request-to-send (RTS) and clear-to-send (CTS) packets to reserve channel and evaluate the signal to interference and noise ratio (SINR) during contention slots. However, long contention periods are not suitable for underwater networks because of the high propagation delay.

\section{SYSTEM MODEL}

In this section, we first briefly describe the unique characteristics of acoustic propagation. Then we introduce an underwater acoustic MIMO transceiver model, and discuss the fundamental multiplexing and diversity tradeoff.

\subsection{Underwater Propagation Model}

Underwater acoustic propagation [30] is substantially different from its RF counterparts [29], which makes QoS delivery of multimedia content a challenging, and largely unexplored, task. Specifically, underwater acoustic communications are mainly influenced by transmission loss, multipath, Doppler spread, and high propagation delay. The transmission loss $T L(d, f)[\mathrm{dB}]$ that a narrowband acoustic signal at frequency $f[\mathrm{KHz}]$ experiences along a distance $d[\mathrm{~m}]$ can be described by the Urick model [30]:

$$
T L(d, f)=\chi \cdot \log (d)+\alpha(f) \cdot d+A .
$$

In (1), the first term account for geometric spreading. The second term accounts for medium absorption, where $\alpha(f)[\mathrm{dB} / \mathrm{m}]$ represents an absorption coefficient. The last term, expressed by the quantity $A[\mathrm{~dB}]$, is the so-called transmission anomaly. More details can be found in $[7,10]$.

\subsection{Acoustic MIMO Transceiver Model}

We consider an underwater acoustic sensor network in which each node has $M_{T}$ transmit elements and $M_{R}$ receive elements (e.g., hydrophones), as shown in Fig. 1. When a node sends information to another node, its bit stream is split into $M_{T}$ sub streams and each sub stream is transmitted by one of the $M_{T}$ transmit elements. All $M_{T}$ transmit elements transmit sub bit streams simultaneously to the receiver with the same carrier frequency and bandwidth. In a narrowband scenario, the received signals at the receiver can be modeled as

$$
Y(t)=\sqrt{P} X(t) H(t)+Z(t)
$$

where $Y(t)=\left[y_{1}(t) y_{2}(t) \cdots y_{M_{R}}(t)\right]$ is the received signal vector whose component $y_{n}(t), 1 \leq n \leq M_{R}$, is the received signal at receive element $n, X(t)=\left[x_{1}(t) x_{2}(t) \cdots x_{M_{T}}(t)\right]$ is the transmitted signal vector whose component $x_{m}(t), 1 \leq m \leq M_{T}$, 
is the transmitted signal from transmit element $m$, and $Z(t)=$ $\left[z_{1}(t) z_{2}(t) \cdots z_{M_{R}}(t)\right]$ is the noise vector whose components are modeled as independent circularly symmetric complex Gaussian random variables with zero mean and unit variance. In (2), $H(t)=$ $\left\{h_{m, n}(t): 1 \leq m \leq M_{T}, 1 \leq n \leq M_{R}\right\}$ is the channel matrix whose component $h_{m, n}(t)$ denotes the channel fading coefficient between transmit element $m, 1 \leq m \leq M_{T}$, and receive element $n, 1 \leq n \leq M_{R}$. We assume that the channel matrix is known at the receiver side, but unknown at the transmitter side. The transmitted signal vector $X(t)$ is assumed to satisfy a power constraint $\sum_{m=1}^{M_{T}}\left|x_{m}(t)\right|^{2}=1$, i.e., the total transmitted power is $P$ no matter how many transmit elements are deployed at the transmit node. Moreover, we assume that the channel is heavily affected by multipath fading (saturated condition, see [22]) as it is often the case in shallow water [2].

\subsection{Multiplexing and Diversity Tradeoff}

The frequency-dependent attenuation significantly limits the maximum usable frequency and thus the available communication bandwidth [5]. MIMO transmissions is thus an ideal way to increase data rates for underwater acoustic communications, in which independent data streams can be sent out in parallel by multiple transmit elements in the same frequency band. The increased spectral efficiency is termed multiplexing gain [33]. At the receiver side, the receiver can demodulate each of the data streams by nulling out the others with a decorrelator [8].

Besides increasing transmission rates, MIMO can also be used to reduce the received signal error probability and hence to improve the communication link reliability. By sending signals that carry the same information through different channels, multiple faded copies of the data information can be obtained at the receiver. Such a redundancy is termed diversity [33] and can be quantified in terms of diversity gain $d$. The average error probability can be reduced in an order of $1 / S N R^{d}$ at high SNR, so the higher the diversity gain, the higher the reliability of the receiver detection.

Therefore, underwater acoustic communications can benefit from MIMO in two aspects: multiplexing gain and diversity gain. Unfortunately, these two gains cannot be optimized independently and there is a tradeoff between them: higher multiplexing gain can be obtained at the price of sacrificing diversity gain, and vice versa. In an RF scenario, for any targeting multiplexing gain $r$, the maximum diversity gain is [33] $d(r)=\left(M_{T}-r\right)\left(M_{R}-r\right)$, which depends on the numbers of transceiver elements $M_{T}$ and $M_{R}$.

\section{UMIMO-MAC}

Let us consider a network of acoustic devices in a multihop environment, and assume that each sensor node is equipped with $M_{T}=M_{R}=M$ transceiver elements. For each packet transmission, each device can encode the information bits to be transmitted in $k$ parallel independent streams, with $k \in 1,2, \cdots, M$. Given the number of independent streams $k$, and given a family of spacetime codes $\mathcal{C}$, a multiplexing gain $r(k)$ and a diversity gain $d(r)$ are defined according to the multiplexing and diversity tradeoff.

Formally, given the number $M$ of transceiver elements at the transmitter and the receiver, and given a set of space time codes $\underline{C}=\left[C_{1}, C_{2}, \cdots C_{P}\right]$, a set of transmission modes $\underline{M}=\left[M_{1}\right.$, $\left.M_{2}, \cdots M_{P}\right]$, with $P$ being the size of the space of transmission modes, are defined between a transmitter and a receiver. Each transmission mode $M_{i}$ is associated to a transmission rate (or simply rate) $R_{i}[\mathrm{bit} / \mathrm{s}]$, with $R_{1} \leq R_{2} \leq R_{P}$, a multiplexing gain $r_{i}$, and a diversity gain $d_{i}$, with the transmission rate increasing with the multiplexing gain, and the bit error rate decreasing with increasing diversity gain.
To explore the relevance of the above decision space to protocol design, let us consider a multimedia application $a$ with bandwidth requirement $\beta^{a}$ and bit error rate $B E R^{a}$. We consider a MIMO CDMA environment [4][6], where a node $i$ needs to transmit a packet to a predetermined neighboring node $j$. To accomplish this, node $i$ needs to: i) limit the near-far effect when it transmits to node $j$; and ii) avoid impairing ongoing communications. These two constraints can be formulated as follows:

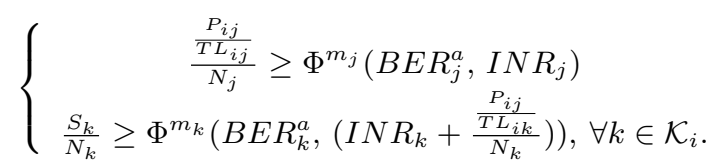

The first inequality in (3) states that the signal-to-noise ratio $\left(S N R_{j}\right)$ at the receiver $j$ needs to be above the SNR threshold $\Phi^{m_{j}}\left(B E R_{j}^{a}\right.$, $\left.I N R_{j}\right)$, i.e., the value that guarantees the bit error rate $B E R_{j}^{a}$ required by the application $a$, given the current interference-to-noise ratio $I N R_{j}$ at the receiver, and a choice of transmission mode $m_{j}$ that determines a multiplexing gain $r_{j}$. The SNR at $j$ is expressed as the ratio between the power received at $j\left(\frac{P_{i j}}{T L_{i j}}\right)$ and the receiver noise $N_{j} . P_{i j}[\mathrm{~W}]$ represents the power transmitted by $i$ to $j$ when an ideal channel (without multipath, i.e., $A=0 \mathrm{~dB}$ ) is assumed, while $T L_{i j}$ and $T L_{i k}$ are the transmission losses from $i$ to $j$ and from $i$ to $k \in \mathcal{K}_{i}$, with $\mathcal{K}_{i}$ being the set of nodes whose ongoing communications may be affected by node $i$ 's transmit power, respectively. Finally, $N_{j}[\mathrm{~W}]$ and $N_{k}[\mathrm{~W}]$ are the noise power at nodes $j$ and $k$, respectively.

The second inequality in (3) represents the same constraint for all transmitters affected by the communication between $i$ and $j$. There, $S_{k}[\mathrm{~W}]$ represents the received power of the signal being decoded by a receiver $k \in \mathcal{K}_{i}$. Note that the interference-to-noise ratio at $k$ is expressed as the sum of the interference-to-noise ratio at $k$ plus an additional component caused by $i$ 's transmission to $j$.

$\Phi^{m}(\cdot)$ depends on the bit error rate and the interference to noise ratio at the receiver. However, the SNR threshold $\Phi^{m}$, as expressed by its dependence on $m$, is also a function of the given choice of transmission mode, i.e., of the multiplexity-diversity tradeoff. Hence, to accommodate the $B E R$ requirements of the application, node $i$ has two choices:

1. For a fixed transmit power $P_{i j}$, use a transmission mode $m$ with multiplexing gain $r$ associated to a SNR threshold $\Phi^{m}(\cdot)$ that is low enough to provide the required $B E R$;

2. For a fixed transmission mode $m$ with multiplexing gain $r$, set its transmit power $P_{i j}$ to the minimum value that guarantees the required $B E R$.

\subsection{The UMIMO-MAC Protocol}

In addition to the objectives previously stated, UMIMO-MAC is designed to reduce the effect of long propagation delays on the channel utilization efficiency, and to efficiently disseminate local information that is needed to make distributed, localized, decisions. We will describe in the following how a suitable transmission mode is selected at the receiver. We refer to Fig. 3, where a transmitter $i$ willing to communicate with a receiver $j$ is depicted. Let us introduce the following:

DEFINITION 1. The upper bound on transmit power $P_{i}^{\max }$ is the maximum transmit power that will not impair ongoing communications for neighbors of transmitter $i$.

DEFINITION 2. The lower bound on transmit power $P_{i j, m}^{m i n}$ is the minimum transmit power needed to decode packet at the receiver $j$ with the required BER for a given transmission mode $m$. 


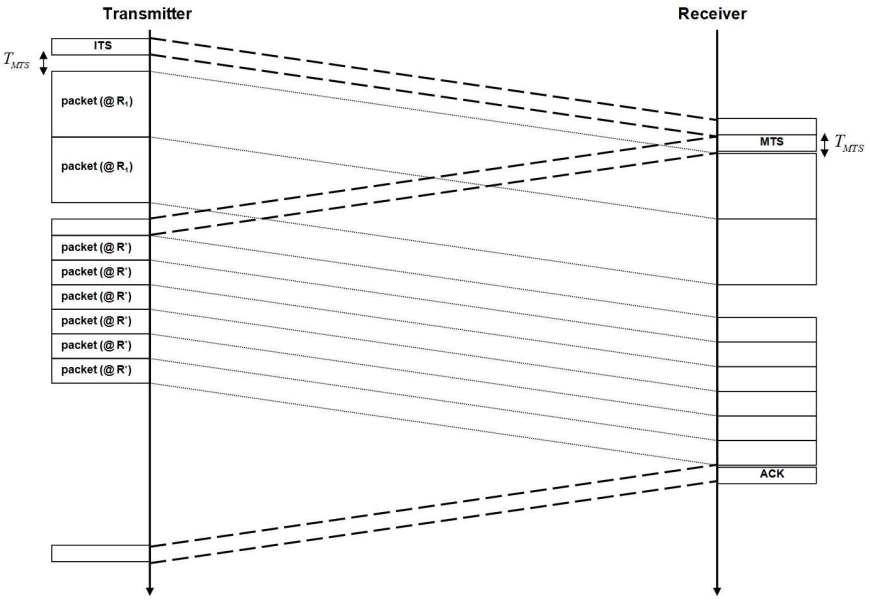

Figure 2: The UMIMO-MAC protocol, where $R_{1}$ is the lowest transmission rate and $R^{*}$ is the assigned transmission rate

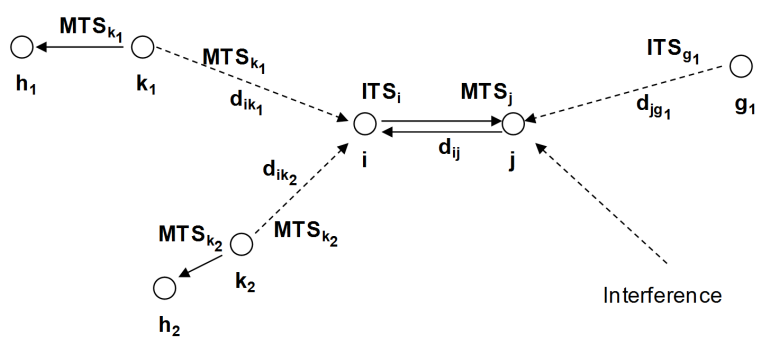

Figure 3: Message transmissions

DEFINITION 3. The assigned transmit power $P_{i j}^{*}$ is the transmit power assigned to transmitter $i$ after negotiation with receiver $j$.

DEFINITION 4. The receiver's interference tolerance $\Delta I_{j}$ is the maximum additional interference that will not impair ongoing communication for receiver $j$.

DEFINITION 5. The finish receive time $t_{j}$ is the time at which receiver $j$ will finish receiving packets whose transmission has already been negotiated.

In UMIMO-MAC, each transmitter $i$ is assumed to know the distance $d_{i j}$ from $i$ to receiver $j$ and the distance $d_{i k}$ from $i$ to $k \in \mathcal{K}_{i}$. Each transmitter $i$ is also assumed to be capable of estimating the transmission loss $T L_{i k}$. Moreover, each receiver $j$ is capable of estimating the multiple access interference (MAI) $I_{j}$, noise power $N_{j}$, distance $d_{i j}$, and transmission loss $T L_{i j}$ between transmitter $i$ and receiver $j$.

Figure 2 illustrates the basic operations and timing of the UMIMOMAC protocol. The protocol employs Intent to Send (ITS) and Mode to Send (MTS) control packets to negotiate and regulate channel access among competing nodes. Note that while this may seem to be analogous to the 802.11-like carrier sense multiple access with collision avoidance protocols (CSMA-CA), the analogy with CSMA-CA is only formal - UMIMO-MAC does not employ carrier sense, and there is no collision avoidance mechanism. In addition, unlike 802.11-like protocols, a single ITS-MTS handshake is used to transmit a block of consecutive packets ${ }^{1}$. This is done to improve

${ }^{1}$ This is in principle allowed also by 802.11 standards, but in practice very seldom used. the utilization efficiency of the underwater channel. ITS and MTS are transmitted using a common spreading code which is known by all nodes.

The ITS contains i) parameters that will be used by the transmitter to generate the spreading code, ii) $P_{i}^{\max }$, the upper bound on transmit power, and iii) the total number of packets that will be transmitted back-to-back. Based on this information, the receiver will be able to locally generate the spreading code that the transmitter will use to send data packets. Based on $P_{i}^{\max }$, the receiver will calculate the appropriate transmit power for the transmitter as will be described in Section 4.4. Besides, by overhearing the ITS, the transmitter's neighbors can become aware of the time when the transmitter will end its transmission.

The MTS contains i) the chosen transmission mode, i.e., the multiplexing and diversity tradeoff, ii) the assigned transmit power $P_{i j}^{*}$, iii) the receiver's interference tolerance $\Delta I_{j}$, and iv) the finish receive time $t_{j}$. The chosen transmission mode and the assigned transmit power will be used by the transmitter to generate the signal. However, power and transmission mode are selected at the receiver, since the latter can be responsive to the dynamics of the channel based on local measurements and consequently control loss recovery and rate adaptation, thus avoiding feedback overheads and latency. The receiver's interference tolerance and finish receive time constitute information intended for the neighbors of the receiver, which will use it to determine their own upper bound on transmission power. Moreover, DATA and ACK are transmitted using the assigned spreading code. ITS, MTS, and ACK are transmitted using the highest diversity gain, i.e., minimum-rate transmission mode, to maximize the probability that they be received correctly.

Before transmitting data, transmitter $i$ overhears ITSs and MTSs from its neighbors. Based on this, the transmitter infers whether its neighbors are involved in other communications; if this is the case, the time at which each neighbor will finish receiving data. Hence, transmitter $i$ decides when to transmit an ITS according to the information overheard in previous ITS or MTS packets. Three scenarios are possible:

1. If no ITS or MTS was received by $i$ from the intended receiver $j, i$ assumes that $j$ is idle, and transmits the ITS immediately;

2. If receiver $j$ recently sent an ITS to a node different from $i$, transmitter $i$ knows that the intended receiver $j$ is currently transmitting data. Transmitter $i$ may or may not know when $j$ will finish transmitting data. If $i$ previously overheard an MTS from the receiver of $j$ 's transmission (for example $g_{1}$ in Fig. 3), then $i$ knows $j$ 's finish transmit time. Otherwise, $i$ can estimate the finish transmit time by assuming that $j$ transmits all packets at the lowest-rate transmission mode.

3. If receiver $j$ previously sent an MTS, transmitter $i$ knows that $j$ is busy receiving data from another node. Hence, $i$ will defer transmission until $j$ 's finish receive time, which it knows since it was contained in the MTS that it overheard.

During the waiting time, transmitter $i$ can potentially receive ITS and MTS packets from its neighbors and update information on ongoing transmissions accordingly. If another node wants to transmit packets to $i, i$ will defer its transmission schedule and receive these packets first. Since the propagation delay is high in underwater, nodes accept packets that have already been transmitted to reduce the channel access delay.

The transmitter does not know the actual interference at the receiver side. Thus, the transmitter can only provide information 
about its upper bound on transmit power to the receiver. As previously mentioned, transmission mode and transmit power are then chosen at the receiver. After transmitting the ITS, instead of just waiting idle for the MTS, which will contain the assigned transmission mode and transmit power, the transmitter starts transmitting packets using the lowest-rate transmission mode data rate. This is done to improve the channel efficiency and thus reduce the effect of the long propagation delays. Immediately after transmitting the ITS, the transmitter waits for $T_{M T S}$ seconds and then transmits packets at the lowest-rate transmission mode with appropriate transmit power, as will be discussed in Section 4.2. $T_{M T S}$ corresponds to the MTS transmission delay plus a turn-around time that is needed by the transceiver electronics to switch between receive and transmit mode, which can be obtained as

$$
T_{M T S}=\frac{L_{M T S} \cdot c}{r_{c}}+T_{e l e c}
$$

where $L_{M T S}$ [bit] is the MTS size, $c$ [bit] is the spreading code length, $r_{c}[\mathrm{chip} / \mathrm{s}]$ is the channel chip rate, and $T_{\text {elec }}$ is the turnaround time needed by the transceiver electronics to switch between receive and transmit mode. Note that $\frac{r_{c}}{c}=R_{1}$, i.e., the lowest-rate transmission mode. Besides, the number of lowest-rate transmission mode packets $n_{L R}$ can be obtained as

$$
n_{L R}=\left\lfloor\frac{\frac{2 \cdot d_{i j}}{\bar{q}}-T_{M T S}}{\frac{L_{D} \cdot c}{r_{c}}}\right\rfloor,
$$

where $\bar{q}$ is the sound velocity and $L_{D}$ [chip/bit] is the packet size. In (5), the time interval that can be used to transmit lowest-rate transmission mode packets is $\left(\frac{2 \cdot d_{i j}}{\bar{q}}-T_{M T S}\right)$, i.e., twice the propagation delay from transmitter $i$ to receiver $j$ minus the time to transmit the MTS, $T_{M T S}$. The transmission delay of a packet at the lowest data rate is $\frac{L_{D} \cdot c}{r_{c}}$. Thus, the number of the lowest-rate transmission mode packets is the round towards minus infinity of $\left(\frac{2 \cdot d_{i j}}{\bar{q}}-T_{M T S}\right) / \frac{L_{D} \cdot c}{r_{c}}$. The receiver will transmit the MTS immediately after receiving the ITS, and the transmitter will transmit packets at the data rate indicated on the MTS at the assigned transmit power immediately after receiving the MTS. After receiving all packets, the receiver will immediately transmit the ACK.

If the transmitter and the receiver are close to each other, i.e., $d_{i j}$ is small, (5) would be zero. The transmitter will start to transmit packets after it receives the MTS when it has 2 or more packets. However, if it has only one packet to send, it will not wait for the MTS. The transmitter will transmit the only packet using the lowest-rate transmission mode, and then wait for the ACK from the receiver. The receiver will still transmit the MTS, but the MTS could only be received by the receiver's neighbors and let them update their information.

\subsection{Upper Bound on the Transmit Power}

As discussed above, the transmitter evaluates its upper bound on transmit power according to local information obtained by overhearing MTSs from its neighbors, as shown in Fig. 3. Thus, the upper bound on transmit power $P_{i}^{\max }$ is calculated by the transmitter as

$$
P_{i}^{\max }=\min \left[P^{\max }, \min _{k \in \mathcal{K}_{i}}\left(\Delta I_{k} \cdot T L_{i k} \mid t_{n o w}+\frac{d_{i k}}{\bar{q}}<t_{k}\right)\right],
$$

where $P^{\max }$ is the maximum transmit power dictated by hardware constraints, $\Delta I_{k}$ is the interference tolerance of node $k \in \mathcal{K}_{i}$. Moreover, $t_{\text {now }}$ is the current time and $t_{k}$ is the finish receive time of node $k \in \mathcal{K}_{i}$.

After transmitting the ITS, the transmitter will transmit packets at the lowest-rate transmission mode until it receives the MTS from the receiver. The transmit power $P_{i j}^{L R}$ used to transmit lowest-rate

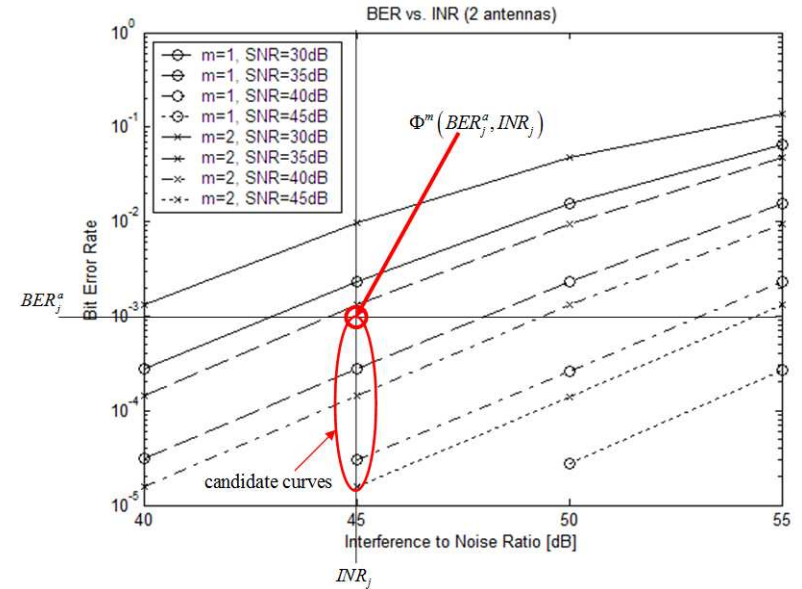

Figure 4: Lower bound SNR threshold example

packets is

$$
P_{i j}^{L R}=P_{i}^{\max }-\Delta P_{t h},
$$

where $\Delta P_{t h}$ is the threshold such that the transmit power will not impair ongoing communications for neighbors, and leave some interference tolerance to them. Since the interference tolerance of the transmitter's neighbors will be very limited if the transmit power is close to the upper bound on transmit power, it is necessary to leave some tolerance for the transmitter's neighbors to overcome additional interference.

\subsection{Lower bound on the Transmit Power}

The receiver evaluates its lower bound on transmit power according to its perceived interference, as illustrated in Fig. 3. The interference to noise ratio (INR) is $I N R_{j}=\frac{I_{j}}{N_{j}}$. Therefore, for a given transmission mode $m, P_{i j, m}^{m i n}$, which is the minimum power needed to decode packet with the required BER, can be obtained as

$$
P_{i j, m}^{m i n}=\Phi^{m}\left(B E R_{j}^{a}, I N R_{j}\right) \cdot T L_{i j} \cdot N_{j} .
$$

Figure 4 graphically illustrates steps and variables involved in calculating $P_{i j, m}^{m i n}$. The plot in Fig. 4 represents the bit error rate (BER) of an underwater acoustic MIMO channel, against varying values of interference-to-noise ratio (on the horizontal axis), for different values of the signal-to-noise ratio and of the transmission mode $m$ (each associated to a multiplexing gain $r)^{2}$. Receiver $j$ estimates the $I N R_{j}$ ( $45 \mathrm{~dB}$ in the figure, indicated by a vertical solid line) and has a target $B E R_{j}^{a}$ of 0.001 (indicated by an horizontal solid line). This defines a set of candidate curves, each of which corresponds to a different allocation of power and choice of a transmission mode, which are able to provide the required target $B E R$ for the given interference conditions. If, for each transmission mode, we set lower bound on transmit power to the value corresponding to the minimum-SNR curve within the set of candidate curves, in the example in the figure we get $\Phi^{1}=32 \mathrm{~dB}$ with $r=1$, and $\Phi^{2}=35.7 \mathrm{~dB}$ with $r=2$. Therefore, once the desired level

\footnotetext{
${ }^{2}$ Note that we consider a rich physical layer model, in which the effects on BER of noise and interference are treated separately. While treating interference as noise is common practice in the networking literature, the peculiar characteristics of underwater communications call for the use of rich physical layer models in protocol design. Hence, in Fig. 4 the bit error rate is plot against varying values of interference-to-noise ratio, for different values of the signal-tonoise ratio.
} 


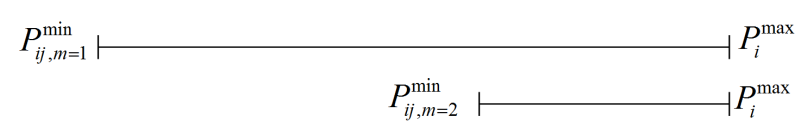

Figure 5: Upper and lower bounds with different choices of transmission mode

of multiplexing gain $r$ is determined (i.e., the transmission mode), the lower bound on the transmit power can be calculated.

\subsection{Joint Selection of Transmission Mode and Transmit Power}

Based on the above discussion, transmit mode and power are selected by the receiver. As shown in Fig. 5, while for different choices of transmission mode the upper bound on transmit power does not vary, the lower bound on transmit power is different, since with a higher diversity gain increases the resiliency to errors and thus allows for a lower transmit power for a given target BER. If the transmit power is chosen to be close to the upper bound on transmit power, the interference tolerance of transmitter neighbors will be very limited. Conversely, if the transmit power is chosen too close to the lower bound, the interference tolerance of the receiver will be very limited. Thus, it is necessary to avoid transmission modes for which upper bound and lower bound on transmit power are too close.

\section{P: Joint Selection of Transmission Mode and Power}

$$
\begin{array}{rc}
\text { Given : } & P_{i}^{\max }, P_{i j, m}^{\min } \\
\text { Find }: & m^{*}, P_{i j}^{*} \\
\text { Maximize }: & \left(\Delta P_{i j, m}=P_{i}^{\max }-P_{i j, m}^{\min }\right) \mid r^{*} \\
\text { Subject to }: & \\
r^{*}=\max \left(r \mid \Delta P_{i j, m}>\Delta P_{t h}\right), \text { or } \\
r^{*}=\min \left(r \mid \Delta P_{i j, m}>\Delta P_{t h}\right) .
\end{array}
$$

The transmit power margin, $\Delta P_{i j, m}=P_{i}^{\max }-P_{i j, m}^{\min }$, evaluates how close are upper and lower bound on transmit power. According to different traffic demands, we choose different levels of multiplexing gain $r^{*}$. For delay-sensitive traffic, e.g., video streams, we choose $r^{*}=\max \left(r \mid \Delta P_{i j, m}>\Delta P_{t h}\right)$ to maximize throughput and decrease delay. For non-real-time data, we choose $r^{*}=\min \left(r \mid \Delta P_{i j, m}>\Delta P_{t h}\right)$ to minimize power consumption and limit interference to neighboring transmissions. After choosing the multiplexing gain, the transmission mode that yields maximum transmit power margin is selected. Then, the assigned transmit power is calculated as $P_{i j}^{*}=P_{i j, m^{*}}^{m i n}+\Delta P_{t o l}$, where $\Delta P_{t o l}$ is a suitable margin. Note that the space of solutions to the above problem is for all practical purposes very limited and can be solved by enumeration - no specialized solver is needed.

\subsection{Interference tolerance and finish receive time}

After selecting transmission mode and transmit power, the receiver can calculate the interference tolerance and finish receive time. For a given transmit power, the signal to noise ratio is $S N R_{j}=$ $\frac{P_{i j}^{*}}{T L_{i j} \cdot N_{j}}>\Phi^{m^{*}}\left(B E R_{j}^{a}, I N R_{j}\right)$. Hence, the receiver has tolerance to overcome additional interference, and the interference tolerance can be obtained as

$$
\Delta I_{j}=\left(\Psi^{m^{*}}\left(B E R_{j}^{a}, S N R_{j}\right)-I N R_{j}\right) \cdot N_{j}
$$

where $\Psi(\cdot)$ is the threshold interference-to-noise ratio, which depends on $m, B E R$, and $S N R$. An example of how to calculate

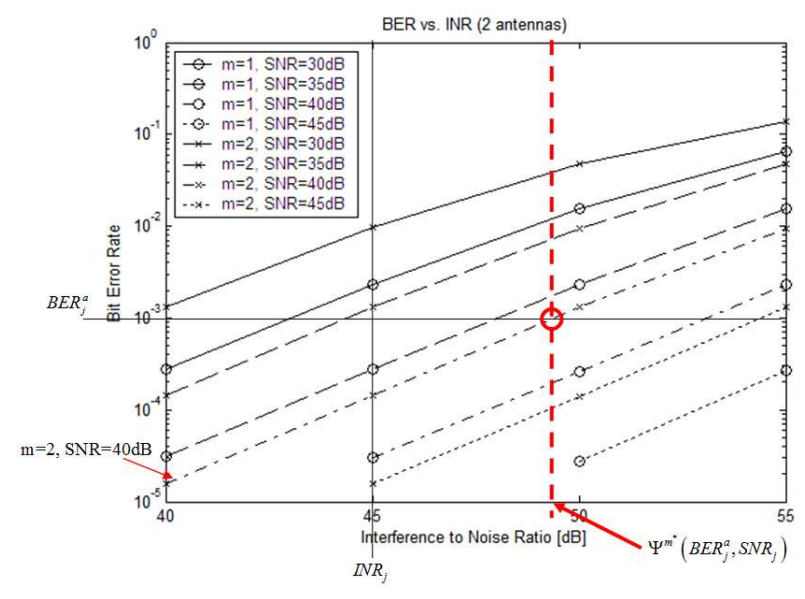

Figure 6: Interference tolerance example

the interference tolerance is shown in Fig. 6. Let us assume that receiver $j$ knows that the selected $m^{*}$ is $2, S N R_{j}$ is $40 \mathrm{~dB}, B E R_{j}^{a}$ is 0.001 , and $I N R_{j}$ is $45 \mathrm{~dB}$. Hence, $\Psi$ needs to be $49.2 \mathrm{~dB}$. The interference tolerance of receiver $j$ can then be calculated according to (11).

The receiver also informs its neighbors of when it will finish receiving packets. According to Fig. 2, the receiver calculates its finish receive time after receiving the ITS. Hence, it calculates the finish receive time $t_{j}$ as

$$
t_{j}=t_{n o w}+T_{M T S}+\frac{2 \cdot d_{i j}}{\bar{q}}+\left(n-n_{L R}\right) \cdot \frac{L_{D} \cdot c}{r^{*} \cdot r_{c}},
$$

where $L_{D}$ [bit] is the packet size, $n$ is the total number of packets that will be transmitted back-to-back, and $n_{L R}$ is the number of lowest-rate transmission mode packets, as in (5). In (12), the first term $t_{\text {now }}$ is the time when receiver $j$ finishes receiving the ITS from transmitter $i$. The second term represents the MTS transmission delay, and the third term accounts for the propagation delay from receiver $j$ to transmitter $i$ and from transmitter $i$ to receiver $j$. The last term represents the transmission delay of the remaining packets. Remember that transmitter $i$ waits for $T_{M T S}$ and then starts transmitting packets using the lowest-rate transmission mode. Hence, some packets are transmitted before transmitter $i$ receives the MTS. Besides, the transmission delay of the assigned transmission mode packet is $\frac{L_{D} \cdot c}{r^{*} \cdot r_{c}}$. Therefore, we can calculate the transmission delay of the remaining packets.

\section{PERFORMANCE EVALUATION}

We have developed a discrete-event object-oriented packet-level simulator to assess the performance of the proposed cross-layer protocol. MIMO links are simulated by incorporating an acoustic MIMO link module, which we have developed to assess MIMO gains on underwater acoustic links. The physical-layer MIMO link module models underwater acoustic signal propagation channel with path loss, Doppler spread, multipath, and underwater delays. The MIMO link module generates bit error rate curves in terms of input parameters such as the link distance, the numbers of transmit/receive elements, choice of space-time codes, total transmit power, acoustic noise level, Doppler spread and correlation among different channels. For example, Fig. 4 is obtained through our underwater MIMO module and represents a comparison of the bit error rate (BER) of an underwater acoustic link, against varying values of interference-to-noise ratio $\left(I N R_{j}=\frac{I_{j}}{N_{j}}\right.$, on the hori- 


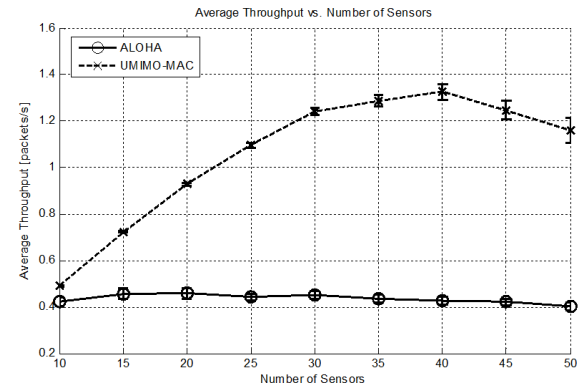

(a)

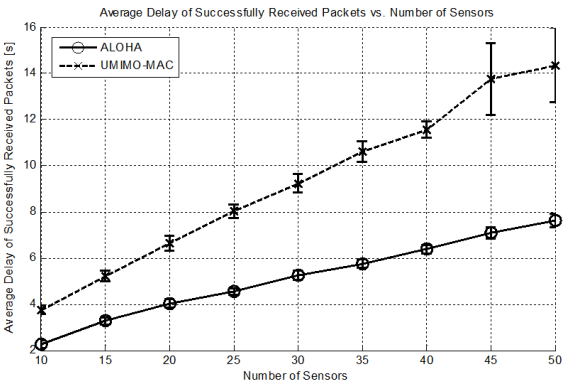

(b)

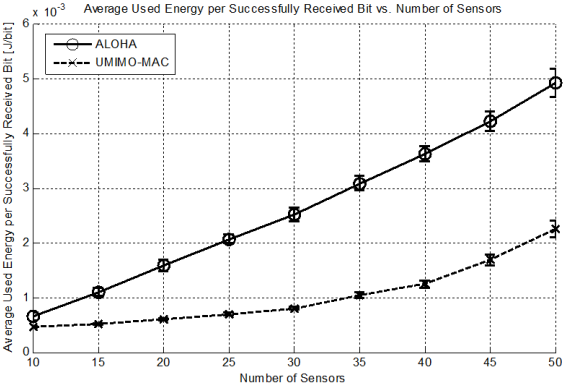

(c)

Figure 7: (a): Average throughput vs. number of sensors; (b): Average delay of successfully received packets vs. number of sensors; (c): Average used energy per successfully received bit vs. number of sensors.

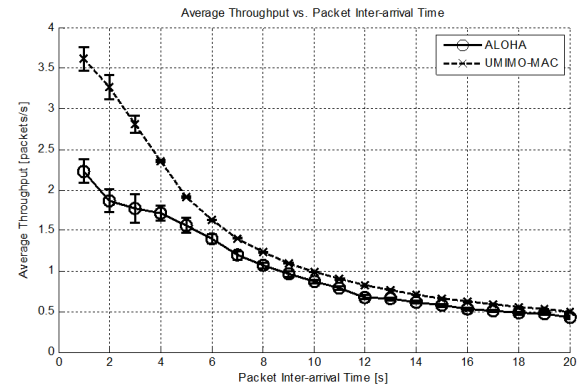

(a)

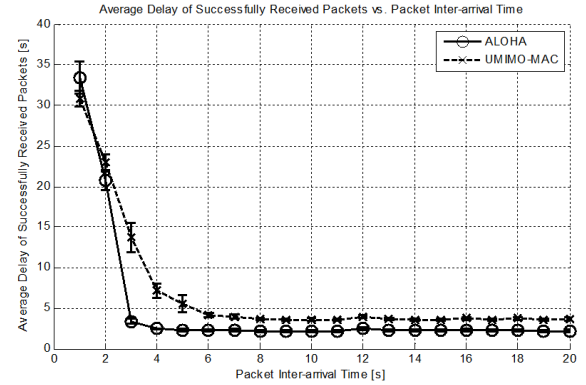

(b)

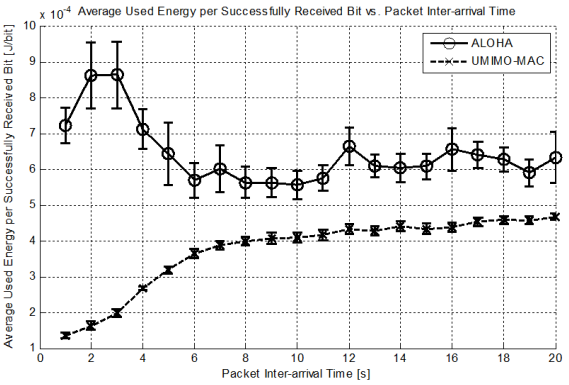

(c)

Figure 8: (a): Average throughput vs. packet inter-arrival time; (b): Average delay of successfully received packets vs. packet inter-arrival time; (c): Average used energy per successfully received bit vs. packet inter-arrival time.

zontal axis), for different values of the signal-to-noise ratio (SNR), with a MIMO diversity, MIMO multiplexing, and SISO system, respectively. We considered a MIMO-CDMA environment $[4,6]$, with fixed length spreading code length 19 , and two transmit and receive antennas. The other simulation parameters are the same as described in [21]. These simulation results, in accordance with preliminary experimental findings in [12, 23, 24, 31], confirm that substantial MIMO gains can be achieved in acoustic channels. We expect that a coding strategy optimized for the underwater acoustic channel will provide even higher performance gains.

We then discuss performance results of UMIMO-MAC and compare it with ALOHA in three-dimensional shallow water. Note that all figures are obtained by averaging over multiple topologies and report $95 \%$ confidence intervals. We set the chip rate $r_{c}$ to $100 \mathrm{kcps}$, the spreading code length $c$ to 19 , the maximum transmission power $P^{\max }$ to $10 \mathrm{~W}$, the data packet size to 250 Bytes, ITS, MTS, and ACK size to 10 Bytes. In addition, we consider an initial node energy of $1000 \mathrm{~J}$, a maximum number of retransmissions equal to 4 , and a queue size of $10 \mathrm{kBytes}$. All deployed sensors are sources and are randomly deployed in the 3D shallow water with volume of $500 \times 500 \times 50 \mathrm{~m}^{3}$. In Fig. 7, we evaluate UMIMO-MAC's scalability and resilience to channel collisions by varying the number of deployed sensors. In Fig. 8, we vary the packet inter-arrival time to measure the effect of traffic.

We set the packet inter-arrival time to $20 \mathrm{~s}$ to avoid queue buffer overflows. When the number of sensors increases, the collision probability increases. In Fig. 7(a), ALOHA is shown to suffer from more collisions and packet retransmissions. Thus, the number of packets dropped after exceeding the maximum number of retransmissions is even higher than the number of successfully received packets. In UMIMO-MAC, only ITS and MTS can collide, and their size is smaller than the packet size. This reduces the collision probability, leading to a higher packet delivery rate.

Fig. 7(b) shows the average delay of successfully received packets. Without two-way handshake, ALOHA can reduce the delay of successfully received packets. However, ALOHA drops a significant amount of packets and its throughput is much lower than UMIMO-MAC. In Fig. 7(c), UMIMO-MAC is shown to considerably reduce the energy consumption (to less than half) by selecting suitable transmit power. Besides, ALOHA consumes a considerable amount of energy in retransmitting packets.

In Fig. 8, we set the number of sensors to 10 to avoid a high number of collisions caused by multiple sensors accessing the channel simultaneously. In Fig. 8(a), the throughput of UMIMO-MAC is shown to be higher than ALOHA even under heavy traffic. The number of dropped packets caused by exceeding the maximum number of retransmissions is still much lower than with ALOHA. However, the queue size is not sufficient to avoid buffer overflows in this heavy-traffic scenario. In this case, UMIMO-MAC drops more buffer overflowed packets than ALOHA in heavy traffic. This is because UMIMO-MAC defers its transmissions if it needs to receive packets that have already been transmitted. However, this problem can be solved by increasing the queue size. With an increased queue size, ALOHA will still drop a significant amount of packets that exceed the maximum number of retransmission threshold.

Finally, in Fig. 8(b), we observe that the average delay of successfully received packets is lower than ALOHA under heavy traf- 
fic conditions. This is because UMIMO-MAC can transmit multiple packets in a train. Moreover, this allows UMIMO-MAC to save a considerable amount of energy, as shown Fig. 8(c).

\section{CONCLUSIONS}

We proposed, discussed and analyzed a medium access control protocol for underwater acoustic sensor networks with MIMO links. UMIMO-MAC adaptively leverages the tradeoff between multiplexing and diversity gain. Moreover, in a cross-layer fashion, UMIMO-MAC jointly selects optimal transmit power and transmission mode through the cooperation of transmitter and receiver to achieve the desired level of reliability and data rate according to application needs and channel condition. UMIMO-MAC was shown to consistently outperform ALOHA in terms of network throughput, average delay and energy consumption under several different simulation scenarios.

\section{REFERENCES}

[1] I. F. Akyildiz, T. Melodia, and K. R. Chowdhury. A Survey on Wireless Multimedia Sensor Networks. Computer Networks (Elsevier), 51(4):921-960, Mar. 2007.

[2] I. F. Akyildiz, D. Pompili, and T. Melodia. Underwater Acoustic Sensor Networks: Research Challenges. Ad Hoc Networks (Elsevier), 3(3):257-279, May 2005.

[3] C. Carbonelli and U. Mitra. Cooperative Multihop Communication for Underwater Acousitc Networks. In Proc. ACM Intl. Workshop on Underwater Networks (WUWNeT'06), September 2006.

[4] R. L. Choi, R. D. Murch, and K. B. Letaief. MIMO CDMA Antenna System for SINR Enhancement. IEEE Trans. Wireless Communications, 2(2):240-249, March 2003.

[5] R. Coates. Underwater Acoustic Systems. John Wiley \& Sons Inc., Hoboken, NJ, 1989.

[6] T. S. Dharma, A. S. Madhukumar, and A. B. Premkumar. MIMO Block Spread CDMA Systems for Broadband Wireless Communications. IEEE Trans. Wireless Communications, 7(6):1987-1992, June 2008.

[7] F. Fisher and V. Simmons. Sound Absorption in Sea Water. Journal of Acoustical Society of America, 62(3):558-564, Sept. 1977.

[8] G. J. Foschini, G. Golden, R. Valenzuela, and P. Wolniansky. Simplified Processing for High Spectral Efficiency Wireless Communication Employing Multi-Element Arrays. IEEE J. Select. Areas on Comm., 17:1841-1852, Nov. 1999.

[9] A. F. Harris and M. Zorzi. On the design of energy-efficient routing protocols in underwater networks. In Proc. of IEEE Intl. Conf. on Sensor and Ad-hoc Communications and Networks (SECON), San Diego, CA, USA, June 2007.

[10] R. Jurdak, C. Lopes, and P. Baldi. Battery Lifetime Estimation and Optimization for Underwater Sensor Networks. IEEE Sensor Network Operations, Winter 2004.

[11] B. W. Ke, Y. J. Zhang, and S. C. Liew. Media Access Control with Spatial Correlation for MIMO Ad Hoc Networks. In Proc. of Intl. Conference on Communications (ICC), pages 3660-3665, Glasgow, June 2007.

[12] D. B. Kilfoyle, J. C. Preisig, and A. B. Baggeroer. Spatial Modulation Experiments in Underwater Acoustic Channel. IEEE Journal of Oceanic Engineering, 30(2):406-415, Apr. 2005.

[13] K. B. Kredo and P. Mohapatra. A Hybrid Medium Access Control Protocol for Underwater Wireless Networks. In Proc. ACM Intl. Workshop on Underwater Networks (WUWNeT'07), September 2007.

[14] Y. H. Kwang, B. Sharif, A. Adams, and O. Hinton. Implementation of multiuser detection strategies for coherent underwater acoustic communication. IEEE Journal of Oceanic Engineering, 27(1):17-27, Jan. 2002.

[15] B. Li, J. Huang, S. Zhou, K. Ball, M. Stojanovic, L. Freitag, and P. Willett. Further Results on High-Rate MIMO-OFDM
Underwater Acoustic Communications. In Proc. of MTS/IEEE OCEANS conference, pages 15-18, Quebec City, Canada, September 2008.

[16] M. Molins and M. Stojanovic. Slotted FAMA: a MAC protocol for underwater acoustic networks. In Proc. of MTS/IEEE OCEANS, Boston, MA, USA, Sept. 2006.

[17] J.-S. Park and M. Gerla. MIMOMAN: A MIMO MAC Protocol for Ad Hoc Networks. Lecture Notes in Computer Science, 3738:207-220, 2005.

[18] D. Pompili and I. F. Akyildiz. A Cross-layer Communication Solution for Multimedia Applications in Underwater Acoustic Sensor Networks. In Proc. of IEEE International Conference on Mobile Ad-hoc and Sensor Systems (MASS), Atlanta, GA, Oct. 2008.

[19] D. Pompili, T. Melodia, and I. F. Akyildiz. A CDMA-based Medium Access Control for Underwater Acoustic Sensor Networks. to appear in IEEE Transactions on Wireless Communications.

[20] D. Pompili, T. Melodia, and I. F. Akyildiz. Routing Algorithms for Delay-insensitive and Delay-sensitive Applications in Underwater Sensor Networks. In Proc. of ACM MobiCom, Los Angeles, LA, USA, Sept. 2006.

[21] D. Pompili, T. Melodia, and I. F. Akyildiz. A Distributed CDMA Medium Access Control for Underwater Acoustic Sensor Networks. In Proc. of Mediterranean Ad Hoc Networking Workshop (Med-Hoc-Net), pages 63-70, Corfu, Greece, June 2007.

[22] J. G. Proakis. Digital Communications. Mc-Graw Hill, New York, 2001.

[23] A. Roy, T. Duman, L. Ghazkhanian, V. McDonald, J. G. Proakis, and J. Zeidler. Enhanced Underwater Acoustic Communication Performance Using Space-Time Coding and Processing. In Proc. of Oceans Conference, volume 1, pages 26-33, Nov. 2004.

[24] H. C. Song, W. S. Hodgkiss, and W. A. Kuperman. MIMO Time Reversal Communications. In Proc. of 2nd Workshop on Underwater Networks, pages 5-10, Montreal, Canada, September 2007.

[25] M. Stojanovic. Recent Advances in High-speed Underwater Acoustic Communications. IEEE Journal of Oceanic Engineering, 21:125-136, Apr. 1996.

[26] M. Stojanovic. On the Relationship Between Capacity and Distance in an Underwater Acoustic Channel. In Proc. ACM Intl. Workshop on Underwater Networks (WUWNeT'06), September 2006.

[27] W. Su and X.-G. Xia. Signal Constellations for Quasi-Orthogonal Space-Time Block Codes with Full Diversity. IEEE Transactions on Information Theory, 50(10):2331-2347, Oct. 2004.

[28] K. Sundaresan, R. Sivakumar, M. A. Ingram, and T.-Y. Chang. Medium Access Control in Ad Hoc Networks with MIMO Links: Optimization Considerations and Algorithms. IEEE Trans. Mobile Computing, 3(4):350-365, Oct.-Dec. 2004.

[29] V. Tarokh, N. Seshadri, and A. R. Calderbank. Space-Time Codes for High Data Rate Wireless Communication: Performance Criterion and Code Construction. IEEE Trans. Inform. Theory, 44(2):744-765, 1998.

[30] R. J. Urick. Principles of Underwater Sound. McGraw-Hill, 1983.

[31] M. Zatman and B. Tracey. Underwater Acoustic MIMO Channel Capacity. In Proc. of 36th Asilomar Conference, volume 2, pages 1364-1368, Nov. 2002.

[32] W. Zhang and U. Mitra. A Delay-Reliability Analysis for Multihop Underwater Acoustic Communication. In Proc. ACM Intl. Workshop on Underwater Networks (WUWNeT'07), September 2007.

[33] L. Zheng and D. N. C. Tse. Diversity and Multiplexing: a Fundamental Tradeoff in Multiple-Antenna Channels. IEEE Transactions on Information Theory, 49(5):1073-1096, May 2003. 